Series editor: David Taylor

\section{How double eyelashes give you swollen legs}

\section{S Jeffery}

\section{The lymphoedema-distichiasis syndrome}

D istichiasis is the presence of aberrant extra eyelashes, and is familiar to adnexal surgeons as these lashes sometimes cause intense irritation of the cornea. This can require removal of the lashes by epilation (plucking), cryotherapy, electrolysis, lid splitting operations, or laser treatment. The lashes are very fine and originate in the meibomian glands at the rear of the tarsal plate. The condition can vary from a few sparse hairs on one lid, to full sets of lashes on both lids. Although individuals are usually aware of the presence of these hairs, quite often they are not. This is probably because of hypoaesthesia of the cornea, and/or outwardly curling lashes.

Lymphoedema is chronic tissue swelling, most frequently of the lower limbs, resulting from deficient lymphatic drainage in the presence of normal capillary function. ${ }^{1}$ Primary lymphoedema is caused by an intrinsic abnormality of the lymph conducting pathways, is genetic in origin, and may be present from birth or can develop later, often at or after puberty. The inheritance of this disorder is autosomal dominant.

At St George's Medical School our group has a long standing interest in the genetics and clinical aspects of the various forms of lymphoedema. "Very interesting," you may possibly think, or alternatively, "so what?" and in any event what is this article doing in a journal of ophthalmology? The answer is in the title, in that there is a specific form of lymphoedema where the swelling of the legs is accompanied by a range of other symptoms, the predominant one being distichiasis. This is at least as common as the lymphoedema itself, and the disorder is therefore known as lymphoedemadistichiasis (LD). In genetic terms the presence of distichiasis is invaluable, as it allows definite phenotyping in a disorder which is known to be both genetically and clinically heterogeneous. We collected a series of families from genetic and dermatology clinics, allowing us to locate the gene for LD on the long arm of chromosome $16 .^{2}$ From there it was possible to identify the gene, which we did, but not before a group in America who were also interested in the lymphoedemas. ${ }^{3}$ They were a nice lot, and of course we weren't bitter, not even a bit! The gene turned out to be FOXC2, a transcription factor involved in numerous developmental pathways, two of which clearly are those of the lymphatics and the associated regions of the eye, as ptosis is also a feature in $30 \%$ of individuals with $\mathrm{LD}^{4}$

\section{We are very interested in finding families with pure distichiasis}

With the discovery of the gene for LD, we wondered whether pure distichiasis was also caused by mutations in FOXC2 We therefore went to an adnexal surgeon, Mr Collin at Moorfields, to find families with this condition. This was successful, as we found three isolated cases and eight families from the records. What we also discovered was that every one of them had associated lymphoedema. Just as it had not been the practice in dermatology clinics to look at the inner eyelid, it was not customary in ophthalmology departments to ask patients to roll up their trouser legs, unless perhaps when performing the mason's handshake. We are very interested in finding families with pure distichiasis, but are wondering whether there are such pedigrees, as every time we are told that there is such a case, they turn out to have lymphoedema. In part, this is a plea to ophthalmologists to help us see whether the situation as it currently exists is correct-that is, that distichiasis occurs only with swollen legs. Finding single individuals with double eyelashes is of interest, although we do know that some cases of LD have only oedema and some only distichiasis (though less than $5 \%$ in each case), but families with distichiasis alone are preferable. If we look and the ophthalmology community looks, we should be able to find the answer.

Now back to the title, which in fact turns out to be misleading. We know the gene for this condition, and that mutations in it produce both distichiasis and swollen legs, but we have no idea how the mutations produce their effect. It is fairly certain that the changes in the FOXC2 gene produce a loss of function of the protein, so that simply having half the amount of the transcription factor impairs the normal developmental pathways. How this affects both lymphatics and the eye is at present a mystery. It also seems, unlike other forms of primary lymphoedema, that there are too many lymphatic vessels produced rather than too few, though those that are present are presumably dysfunctional. Why is the distichiasis present from birth, while the oedema usually comes on at puberty or later? Once again, we don't know, though the effect of hormones may play a part. If we are able to analyse families with just distichiasis, or just ptosis, and identify the genes underlying these conditions, it might well be that these act in the pathways controlled by FOXC2, and their discovery could help to unravel these developmental processes.

Br J Ophthalmol 2002;86:1074

\section{Author's affiliations}

S Jeffery, Medical Genetics Unit, St George's Hospital Medical School, Cranmer Terrace, Tooting, London SW17 ORE, UK;

s.jeffery@sghms.ac.uk

Accepted for publication 1 May 2002

\section{REFERENCES}

1 Mortimer PS. Managing lymphoedema. Clin Dermatol 1995:13:499-505.

2 Mangion J, Rahman N, Mansour S, et al. A gene for lymphedema-distichiasis maps to 16q24.3. Am J Hum Genet 1999;65:427-32.

3 Fang J, Dagenais SL, Erickson RP, et al. Mutations in FOXC2 (MFH-1), a forkhead family transcription factor, are responsible for the hereditary lymphedema-distichiasis syndrome. Am J Hum Genet 2000;67 1382-8.

4 Brice G, Mansour S, Bell R, et al. Analysis of the phenotypic abnormalities in lymphoedema distichiasis syndrome in 74 patients with FOXC2 mutations or linkage to 16q24. J Med Genet 2002; (in press). 